\title{
¿Qué interpelamos cuando pensamos la ciudad? Fundaciones, proyectos y preguntas de investigación en la obra de Chicago
}

\author{
What we interpel when think the city? Foundations, projects and \\ research questions at the work of Chicago
}

Ana Laura Hidalgo hidalgo.analaura@gmail.com

http://orcid.org/0000-0002-6900-5120

Facultad de Ciencias Humanas; Universidad Nacional de San Luis/ Consejo Nacional de Investigaciones Científicas y Técnicas (Argentina)

\section{Resumen}

El artículo recupera la tradición de los clásicos de la Escuela de Chicago para pensar en preguntas de investigación acerca de la ciudad como objeto de estudio. Ante la imposibilidad de considerar una historia lineal, se presentan tres fundaciones diferentes que dieron lugar a la delimitación del objeto de estudio de los investigadores del Departamento de Sociología de la Universidad de Chicago de principios del siglo XX. Esto condiciona la diversidad de proyectos de los clásicos, proponiendo una relectura de la obra de Park.

Palabras clave: ciudad; Escuela de Chicago; Park. 
Abstract

The article recovers the tradition of the classics of the Chicago School to think about research questions about the city as an object of study. Given the impossibility of considering a linear history, three different foundations are presented that led to the delimitation of the object of study of the researchers of the Department of Sociology of the University of Chicago at the beginning of the 20th century. This conditions the diversity of projects of the classics, proposing a re-reading of Park's work.

Keywords: city; Chicago School; Park.

El estudio de los procesos sociales que tienen lugar en las ciudades admiten considerar diversos abordajes disciplinares; la sociología, la economía, la comunicación, la ciencia política, la antropología, entre otras, elaboran interrogantes que atraviesan parte de las problemáticas que tienen lugar en las áreas urbanas con alta concentración de personas.

Si bien el término "sociología urbana" comenzó a difundirse en 1910 en los Estados Unidos, Christian Topalov considera que no es posible reconocer una historia lineal de llamada sociología urbana en relación a los fundadores clásicos o problemas abordados por esta disciplina.

\begin{abstract}
Esta disciplina no ha experimentado un proceso de acumulación de saberes en el marco de una institucionalización estable, sino más bien una serie discontinua de surgimientos locales, seguidos por momentos de oscurecimiento. La definición de su objeto de estudio ha cambiado con frecuencia, a veces de manera radical. La razón de ello es que cada una de esas definiciones se relaciona con la configuración histórica particular del momento y el país en cuestión, pese a algunos episodios de internacionalización parcial (Topalov, 2012:
\end{abstract} 1).

En su conferencia titulada Treinta años de sociología urbana. Un punto de vista francés señala que en Sociologie urbaine (1968), Ledrut (1) considera que las intervenciones del urbanismo cuestionan la organización y el funcionamiento del espacio social; por tanto, requiere de la mirada de los sociólogos en este proceso. "La planificación urbana es "un medio de control social del orden urbano", un medio de "controlar la transformación de la ciudad". El sociólogo se ofrece entonces para ayudar a ordenar las ciudades de manera racional. La ciudad debe ser 
un lugar de integración social, y la sociología urbana debe estudiar las condiciones de cohesión de las unidades que componen el espacio: su cohesión interna -se necesitan "auténticos barrios"- y la cohesión del todo que forma la aglomeración" (Topalov, 2012: 3). El trabajo teórico de Ledrut recupera argumentaciones de la literatura producida a principios del siglo XX en Estados Unidos; esto le permitió validar científicamente esta disciplina, al tiempo que invisibilizar a sus antecesores franceses; Chombart de Lauwe (2) y Lefebvre (3). Topalov (2012) reconoce en el manual de Ledrut una intención de fundar una sociología urbana al servicio de una planificación mejor informada y más humana.

Sin embargo, los franceses no fueron los primeros en preguntarse por estos procesos.

\section{La Escuela de Chicago}

Topalov (2004) señala tres diversas acciones que originaron tres fundaciones de la llamada Escuela de Chicago. Sin embargo, entre 1920 y 1930, los sociólogos del Departamento de Sociología de esa universidad no se consideraban miembros de una "escuela", ni siquiera los del grupo más reducido que giraba en torno a Park y Burgess. La etiqueta fue atribuida "a profesores y estudiantes, temas y conceptos, investigaciones y publicaciones ligados al departamento de Sociología de la Universidad de Chicago en su "edad de oro", entre 1920 y 1932 -si adoptamos la cronología propuesta por Robert E. L. Faris (1970)" (Topalov, 2004: 1).

Advierte que esto comienza a cambiar hacia la década de 1960, en el cual la Escuela de Chicago había empezado a ser mencionada de modo frecuente. No obstante, eran "tres acciones, en tres momentos distintos originaron tres 'Escuelas de Chicago', que sólo tenían en común el nombre" (Topalov, 2004: 3).

Reconoce tres momentos centrales que dieron lugar a la delimitación del objeto de estudio de los investigadores del Departamento de Sociología de la Universidad de Chicago de principios del siglo XX. El primero de ellos es un encuentro con trabajadores sociales, planificadores urbanos y actores del ámbito privado realizado en Toronto en 1923; el eje de trabajo fue "escenario y vida cotidiana". Esta reunión permitió el encuentro de tres mundos que dialogaban entorno al vecindario, aun cuando sus proyectos no eran iguales.

El segundo momento de invención fue un acontecimiento realizado ese mismo año en la Universidad de Chicago denominado Local Communit Research Comunite, en el cual se promovía la investigación de temas locales; este evento fue financiado por la Fundación Rockefeller. Se destacaban por abordar problemas sociales sobre el vecindario en los cuales confluye una base empírica y una mirada científica. 
El tercer acontecimiento lo llama "conversación"; este se produjo entre los sociólogos de la Escuela de Chicago y el conjunto de sociólogos de EE. UU. en 1925 en una Reunión de la Sociedad Sociología Americana.

Por tanto, los sociólogos del Departamento de la Escuela de Chicago que desarrollaron su trabajo a principios del siglo XX se caracterizaron por su problematizaciones entorno a la ciudad y los actores urbanos. Esta periodización nos invita a reflexionar y (re)leer a los clásicos que problematizaron la noción de ciudad y se permitieron reconocer en ella la incidencia de diversas categorías de análisis. En este sentido, este artículo continúa por un trazado por un fragmento de la obra de Park The City, publicada por primera vez en 1915.

\section{El trabajo de Park. Una relectura}

En la introducción a la reconocida obra de Robert Ezra Park La ciudad y otros ensayos de ecología humana, Emilio Martínez señala que existe una razón -entre otras-, de "índole teórica para regresar a ellos [los clásicos]: rescatar categorías, conceptos y argumentos todavía útiles para comprender la realidad social" (Park, 1999: 8). Robert E. Park (1864-1944) fue un sociólogo de la Escuela de Chicago quien se caracterizó por su esfuerzo por llevar a la institucionalización la disciplina de la sociología de la ciudad.

Pizorno reconoce en el trabajo de Park aportes teóricos de los trabajos de Simmel y Durkheim; sus fundamentaciones se asientan en la investigación empírica, y "se articula a través de un sencillo modelo de explicación de los fenómenos sociales (y urbanos, en particular)" (Park, 1999: 9).

Martínez (1999) argumenta que el trabajo de Park no se caracteriza tanto por su consistencia teórica o síntesis, como por los instrumentos analíticos que propone en la "ecología humana" (4). En los límites de una comunidad particular, operan relaciones de fuerza que tienden a otorgar a la misma un orden social; Park utiliza la noción de "ecología humana" para denominar a la ciencia que aborda esos agrupamientos y las constelaciones situadas entre las personas e instituciones que se producen en la convergencia de esas fuerzas.

\section{El objeto de estudio: la ciudad}

La sociología urbana se pregunta frecuentemente ¿cómo definir la especificidad de su objeto? (Topalov, 2012). Si bien esta pregunta admite numerosas respuestas, en los acontecimientos 
fundacionales llevados a cabo por los investigadores de Chicago que describimos precedentemente, se puede reconocer como objeto privilegiado, la ciudad.

En "La ciudad: sugerencias para la investigación del comportamiento humano en el medio urbano", Park la define como:

\begin{abstract}
un estado de ánimo, un conjunto de costumbres y tradiciones, de actitudes organizadas y de sentimientos inherentes a esas costumbres, que se transmiten mediante dicha tradición. En otras palabras, la ciudad no es simplemente un mecanismo físico y una construcción artificial: está implicada en los procesos vitales de las gentes que la forman; es un producto de la naturaleza y, en particular, de la naturaleza humana (Park, 1999: 49).
\end{abstract}

Por su parte, Burgess (1925) señala que en la ciudad conviven una variedad amplia de actores, con racionalidades particulares e intereses diversificados. Por tanto, la ciudad es un espacio en el cual las relaciones sociales se encuentran en acuerdos y desacuerdos en la vida cotidiana en el ambiente urbano; es una unidad geográfica, ecológica y económica, que se funda en la división del trabajo; es por tanto, "como un todo orgánico; una especie de mecanismo psicofísico en el cual y a través del cual, los intereses privados y políticos encuentran una expresión no sólo colectiva sino además organizada" (Park, 1999: 50).

Park reconoce contribuciones centrales que permiten situar sus abordajes relativos a sus métodos de observación social acerca de los problemas urbanos de su contemporaneidad; la vida y la cultura urbanas del hombre civilizado, son un objeto de investigación que puede ser abordado con los mismos métodos de observación que la antropología se ha dedicado a estudiar a los pueblos primitivos.

El texto de Park define un punto de vista sobre un posible programa de estudio de la vida urbana, que caracteriza como "plano de la ciudad y organización formal". En él sostiene que la ciudad está arraigada en los hábitos y costumbres de las personas que la habitan; por tanto, se encuentra caracterizada tanto por una organización material como por una organización moral, y sus interacciones permiten una adaptación mutua entre ambos aspectos; en este sentido, sostiene que la forma geométrica de la ciudad es una construcción artificial.

Esta interacción es tal que la estructura de la ciudad se fundamenta en la naturaleza humana; pero simultáneamente, la organización de la ciudad se impone a los sujetos como un "dato bruto y exterior que les modela según propósitos e intereses propios" (Park, 1999: 51).

De este modo, el carácter del entorno urbano y la organización moral que conlleva se encuentran determinados por el tamaño de la concentración y distribución de la población en 
un área urbana particular. Por otra parte, la organización de la vida urbana genera relaciones de proximidad que son posibilitados por los contactos de vecindad.

El vecindario existe sin organización formal. La sociedad de desarrollo local es una estructura erigida sobre la base de la organización espontánea de los vecinos y se constituye con el propósito de dar expresión al sentimiento local sobre aquello que atañe a los intereses locales (Park, 1999: 54).

Un segundo punto que desarrolla, hace referencia a "la organización industrial y el orden moral" de la ciudad. Ahí trabaja una distinción entre la ciudad antigua y la moderna, caracterizando a esta última como

\begin{abstract}
una plaza de comercio y debe su existencia al mercado alrededor del cual se desarrolla. La competencia industrial y la división del trabajo, que con toda probabilidad han contribuido en gran medida al desarrollo de las energías latentes de la humanidad, sólo son posibles por la existencia de los mercados, del dinero y de otros medios que facilitan los negocios y el comercio (Park, 1999: 57)
\end{abstract}

Por tanto, la ciudad está constituida por relaciones sociales basadas en la división del trabajo; que permitieron un incremento de la interdependencia de los sujetos. El individuo depende de otros integrantes de la comunidad que integra; de modo que "este incremento de la interdependencia entre las partes ocasiona en la organización industrial general un cierto tipo de solidaridad social basada no ya en el sentimiento y en el hábito, sino en la comunidad de intereses" (Park, 1999: 60). Siguiendo lo desarrollado por Smith en La riquezas de las naciones, reconoce los procesos de especialización del trabajo y la búsqueda individual del desarrollo de aptitudes aptas para la competencia. Con esto, el desarrollo de escuelas comerciales y agencias de orientación profesional de modo que los individuos desarrollen aptitudes para la competencia que genere un "equilibrio inestable".

A lo anterior, se suma el proceso de movilidad de la población asociado a la búsqueda del empleo al cual pueden postularse a calificar. De este modo, "la movilidad de un individuo o de una población no se mide únicamente por el cambio de localización, sino también por el número y la diversidad de estímulos a los que uno y otra responden" (Park, 1999: 61).

Park argumenta que la concentración de población en las áreas urbanas, los diversificados mercados, la división del trabajo, la concentración de individuos y grupos en tareas específicas, 
permitieron la modificación de las condiciones materiales de la vida, haciendo cada vez más necesarios transformaciones de acuerdo a las nuevas condiciones.

El tercer punto que desarrolla, alude a las "elaciones secundarias y control social". En él desarrolla la incidencia de los medios de comunicación y de transporte urbanos de su época. Sus transformaciones permitieron cambios no sólo en la organización industrial, sino también en la distribución de la población. Consecuentemente, fueron modelando nuevos hábitos, sentimientos y caracteres en la población urbana.

En este mismo apartado, Park desarrolla otras nociones que considera de importancia relevante en el proceso de estudio de lo urbano. Considera a "la política de los partidos y la publicidad" y "la publicidad y el control social". Diferencia en este punto dos formas de organización social; una basada en las relaciones personales o primarias, y otra que apelan al público o relaciones secundarias que en general no se conocen personalmente. "El aparato político procura el control sobre un grupo primario" (Park, 1999: 75). La publicidad, en tanto, permite ser reconocida como una forma de control social, y desempeña un rol relevante en sociedades caracterizadas por relaciones secundarias. "En los grupos secundarios y en la ciudad, la moda tiende a reemplazar la costumbre, y la opinión pública pasa a ser, antes que las costumbres, un factor dominante del control social" (Park, 1999: 78). En este sentido, ubica a la prensa como el más importante de esos instrumentos de control social.

El cuarto punto que presenta Park, alude a "El temperamento y el medio urbano"; en él desarrolla los derivados que considera de la convivencia en las ciudades de razas y culturas diferentes. De este modo, los transportes, las comunicaciones, y la segregación de la población urbana tiende a facilitar la movilidad de los individuos.

\begin{abstract}
Los procesos de segregación instauran distancias morales que convierten la ciudad en un mosaico de pequeños mundos que se tocan sin llegar a penetrarse. Esto hace posible que los individuos pasen rápida y fácilmente de un medio moral a otro y alienta la fascinante aunque peligrosa experiencia de vivir al mismo tiempo en mundos diferentes y contiguos, pero por lo demás completamente separados. Todo eso tiende a conferir a la vida urbana un carácter superficial y casual, a complicar las relaciones sociales, y a producir nuevos y divergentes tipos de individuos. Esto introduce al mismo tiempo un elemento de azar y de aventura que se añade a la excitación de la vida urbana y le otorga un atractivo particular para los temperamentos jóvenes y fogosos (Park, 1999: 80).
\end{abstract}

Park vincula la vida urbana con otra moralidad propia de la concentración y diversificación que la caracteriza. Así, "la población tiende por sí misma a segregarse, no sólo en virtud de sus 
intereses, sino también de acuerdo con sus gustos y sus temperamentos [...]. Cada barrio pueda asumir el carácter de una región moral" (Park, 1999: 81).

\section{Palabras finales}

Topalov señala la inestabilidad de la disciplina, de sus instrumentos de análisis y de los objetos de estudio que privilegia; y lo hace reconociendo la mirada situada a partir de la cual interpreta a la sociología urbana. Este fue el punto de partida a partir del cual nos acercamos a una (re)lectura de un fragmento de la obra de un referente ineludible de la Escuela de Chicago.

El trabajo de Park que sistematizamos en esta lectura nos permitió organizar abanicos de posibles abordajes de las problemáticas de las áreas urbanas. Esta aproximación no conlleva la pretensión de agotar el recorrido por el fragmento de la obra de Park escogido; sino por el contrario situar estos posibles caminos de tránsito por su obra, en el contexto de la tradición de la primera generación de la llamada Escuela de Chicago.

Un sendero que nos conduce nuevamente a preguntarnos por el objeto de estudio de la sociología urbana; por sus fundaciones, preguntas y proyectos. Pero fundamentalmente, nos interrogamos ¿por qué la ciudad es un objeto válido para producir conocimiento desde la comunicación?:

Por las oportunidades que ofrece, en particular a los tipos humanos excepcionales o anormales, una gran ciudad tiende a desplegar y a descubrir ante el público, de forma masiva, todos los rasgos y caracteres de la naturaleza humana normalmente oscurecidos y contenidos en las comunidades más pequeñas. En una palabra, la ciudad muestra el bien y el mal de la naturaleza humana, pero de manera excesiva. Quizás esto, mejor que otra cosa, justifica la idea según la cual la ciudad es un laboratorio o clínica donde la naturaleza humana y los procesos sociales pueden ser oportuna y provechosamente estudiados (Park, 1999: 83).

La invitación es a aproximarnos a la relectura de los clásicos, los primeros que problematizaron la noción de ciudad para generar preguntas de investigación situadas a nuestros horizontes de interpretación particulares. 


\section{Notas}

(1) Ledrut había defendido en 1966 una tesis dirigida por Gorges Gurvitch, otro profesor de la Sorbona, titulada Sociologie urbaine et aménagement urbain (Sociología y desarrollo/ordenamiento urbano).

(2) Chombart se desempeñaba desde 1960 como director de investigación en la École Pratique des Hautes Études que luego sería la École des Hautes Études en Sciences Sociales-, institución más bien marginal en esa época.

(3) Henri Lefebvre era profesor de sociología en la Facultad de Letras de Nanterre. Había escrito sobre "la vida cotidiana" y sobre los grandes conjuntos urbanísticos, antes de publicar en 1968 el manifiesto Le droit à la ville (EI derecho a la ciudad).

(4) La noción de "ecología humana" es un concepto que pretendía abarcar la gran metrópoli; a diferencia de la "ecología urbana" de invención francesa.

\section{Bibliografía}

Burgess, E. (1925). El crecimiento de la ciudad: Introducción a un proyecto de investigación. En Park, R.; Burgess, E. y McKenzie, R. The City. Chicago y Londres: The University of Chicago Press.

Martínez, E. (1999). Introducción. En Park, R. E. La ciudad y otros ensayos de ecología urbana. Estudio preliminar y traducción de Emilio Martínez (pp. 49-83). Barcelona: Del Serbal.

Park, R. E. (1999). La ciudad: sugerencias para la investigación del comportamiento humano en el medio urbano. En Park, R. E. La ciudad y otros ensayos de ecología urbana. Estudio preliminar y traducción de Emilio Martínez. Barcelona: Del Serbal.

Topalov, C. (2004). Les usages stratégiques de l'histoire des disciplines. Le cas de l'école de Chicago' en sociologie. In Heilbron, J.; Lenoir, R. et Sapiro, G. (ed.). Pour une histoire des sciences sociales. Hommage à Pierre Bourdieu (pp. 127-157). Paris: Fayard, 2004.

Topalov, C. (noviembre de 2012). Treinta años de sociología urbana. Un punto de vista francés. Conferencia pronunciada en Buenos Aires en la inauguración del Posgrado de Estudios Urbanos, Universidad Nacional de General Sarmiento. 